\title{
Erratum: Growth of conformal graphene cages on micrometre-sized silicon particles as stable battery anodes
}

Yuzhang Li, Kai Yan, Hyun-Wook Lee, Zhenda Lu, Nian Liu and Yi Cui

Nature Energy 1, 15029 (2016); published 25 January 2016; corrected 1 February 2016.

In the version of this Article originally published, Fig. 5b contained a typographical error and the separation between the dashed lines in the right panel should have been labelled $17.2 \mu \mathrm{m}$. This has been corrected 1 February 2016. 